\title{
Perfil Clínico e Evolução de Pacientes com Infecção Relacionada a Dispositivos Cardíacos Eletrônicos Implantáveis
}

\author{
Clinical Profile and Outcome of Patients with Cardiac Implantable Electronic Device-Related Infection
}

\author{
Alessandra de Souza Maciel ${ }^{10}$ e Rose Mary Ferreira Lisboa da Silva ${ }^{2}{ }^{\circledR}$ \\ Universidade Federal de Minas Gerais - Hospital das Clínicas, ${ }^{1}$ Belo Horizonte, MG - Brasil \\ Universidade Federal de Minas Gerais - Departamento de Clínica Médica, ${ }^{2}$ Belo Horizonte, MG - Brasil
}

\section{Resumo}

Fundamento: Houve aumento expressivo na incidência de infecções relacionadas a dispositivos cardíacos eletrônicos implantáveis (DCEI) nos últimos anos, com impacto na mortalidade.

Objetivos: Verificar a proporção de pacientes com infecção de DCEI e analisar seu perfil clínico, as variáveis relacionadas com a infecção e sua evolução.

Método: Estudo retrospectivo, observacional e longitudinal com 123 pacientes com infecção de DCEI entre 6.406 procedimentos. Foram usados os testes paramétricos, e o nível de significância adotado na análise estatística foi de $5 \%$.

Resultados: A idade média dos pacientes foi de 60,1 anos, e 71 eram homens. A média de internação foi de 35,3 dias, e houve remoção total do sistema em 105 pacientes. Identificaram-se endocardite infecciosa (EI) e sepse em 71 e 23 pacientes, respectivamente. A mortalidade intra-hospitalar foi 19,5\%. Houve associação entre El e extrusão do gerador $(\mathbf{1 7 , 0} \%$ vs. $19,5 \%$ nos grupos com e sem El, respectivamente, $p=0,04$; associação inversa) e sepse $(15,4 \%$ vs. 3,2\%, $p=0,01)$. Houve associação entre morte intra-hospitalar e EI $(83,3 \%$ vs. $52,0 \%$ com e sem morte, respectivamente, $p=0,005)$ e sepse $(62,5 \%$ vs. $8,1 \%, p<0,0001)$. Foi dada alta hospitalar a 99 pacientes. Durante a média de seguimento clínico de 43,8 meses, a taxa de mortalidade foi de $43 \%$, e $65,2 \%$ dos pacientes com sepse faleceram (p $<0,0001$ ). A curva de sobrevida de Kaplan-Meier não indicou associação significante com sexo, agente etiológico, fração de ejeção, El e modalidade de tratamento. A taxa de mortalidade foi de $32,8 \%$ entre os pacientes submetidos a reimplante de eletrodos por via endocárdica e $52,2 \%$ entre aqueles por via epicárdica $(p=0,04)$. Não houve influência da etiologia chagásica, a qual correspondeu a $44,7 \%$ das cardiopatias de base, quanto às variáveis clínicas e laboratoriais ou à evolução.

Conclusões: A taxa de infecção foi de 1,9\%, com predomínio em homens. Houve associação entre mortalidade intrahospitalar e El e sepse. Após a alta hospitalar, a taxa de mortalidade anual foi de 11,8\%, com influência de sepse durante a internação e o implante epicárdico.

Palavras-chave: Marca-Passo Artificial; Procedimentos Cirúrgicos Cardiovasculares; Bactéria; Endocardite; Evolução Clínica; Infecção.

\footnotetext{
Abstract

Background: In recent years, the incidence of infections related to cardiac implantable electronic devices (CIED) has increased sharply, impacting mortality.

Objective: To verify the proportion of patients with CIED infection; to analyze their clinical profile and the variables related to the infection and its progression.

Methods: Retrospective and longitudinal observational study including 123 patients with CIED infection among 6406 procedures. Parametric tests and a level of significance of $5 \%$ were used in the statistical analyses

Results: The mean age of patients was 60.1 years and mean length of stay in hospital was 35.3 days; most (71) patients were male, and the system was completely removed in 105 cases. Infectious endocarditis (IE) and sepsis were observed in 71 and 23 patients, respectively. Intrahospital mortality was $19.5 \%$. IE was associated with extrusion of the generator $(17.0 \%$ vs $19.5 \%$ with and without IE, respectively, $p=0.04$, inverse association) and sepsis (15.4\% vs 3.2\%, $p=0.01)$. Intra-hospital death was associated with IE $(83.3 \%$ vs $52.0 \%$ with and without intrahospital death, respectively, $p=0.005)$ and sepsis $(62.5 \%$ vs $8.1 \%, p<0.0001)$. Ninety-nine patients were discharged. During a mean follow-
}

Correspondência: Rose Mary Ferreira Lisboa da Silva •

Universidade Federal de Minas Gerais - Cardiologia - Avenida Alfredo Balena, 110. CEP 30130-100. Belo Horizonte, MG - Brasil

E-mail: roselisboa@uol.com.br

Artigo recebido em 21/08/2019, revisado em 30/03/2020, aceito em 16/06/2020

DOI: https://doi.org/10.36660/abc.20190546 
up of 43.8 months, mortality rate was 43\%; among patients with sepsis, it was 65.2\% (p < 0.0001). By applying a Kaplan-Meier survival curve, we did not indicate significant associations with sex, etiologic agent, ejection fraction, IE, or treatment modality. The death rate was $32.8 \%$ for patients subjected to endocardial electrode reimplantation and $52.2 \%$ for epicardial reimplantation ( $p=0.04)$. Chagasic etiology (44.7\% of the baseline heart diseases) did not influence clinical and laboratory variables or disease progression.

Conclusion: The infection rate was 1.9\%, mostly in men. We observed an association of intra-hospital mortality with IE and sepsis. After discharge, the annual mortality rate was $11.8 \%$, influenced by sepsis during hospitalization and epicardial implantation.

Keywords: Cardiac Pacemaker Implantation; Cardiovascular Surgical Procedures; Bacteria; Endocarditis; Outcome; infection.

Full texts in English - http://www.arquivosonline.com.br

\section{Introdução}

O uso de dispositivos cardíacos eletrônicos implantáveis (DCEI) cresceu de forma exponencial nos últimos 10 anos devido ao avanço da tecnologia, à ampliação das indicações e à maior expectativa de vida. Por outro lado, durante esse período, houve um aumento importante e desproporcional (de 210\%) na incidência de infecções relacionadas aos DCEI, alcançando a incidência de até 19,9\%. ${ }^{1-4}$ Essas infecções estão relacionadas ao tipo de dispositivo e ao número de suas manipulações. ${ }^{5}$ Após a realização de substituição do dispositivo, o risco de infecção é de cerca de 5\%, com um aumento de 2 a 4 vezes em comparação com o risco de um implante primário., ${ }^{5,6}$ Outros fatores também estão associados com o aumento da infecção, tais como sexo, idade, comorbidades e falta de profilaxia. ${ }^{7,8}$

A infecção relacionado com DCEI apresenta morbidade significativa e mortalidade intra-hospitalar que varia de 6 a $14 \%$, com mortalidade total de aproximadamente $20 \%$ em um ano, incluindo o período após a alta hospitalar. ${ }^{1,6,9}$ Além disso, há variáveis associadas a desfechos desfavoráveis e preditores de mortalidade, como idade do paciente, uso de marca-passo (MP) temporário, trocas de dispositivos, agente estafilococo como etiologia, presença de prótese valvar cardíaca, tempo de remoção do dispositivo, insuficiência renal, necessidade de transfusão de sangue e presença de endocardite. ${ }^{1,10-13} \mathrm{O}$ risco de morte por infecção do DCEI depende do tipo de dispositivo e persiste ao longo do tempo. A taxa de mortalidade de até $20 \%$ permanece durante 3 anos para MP de câmera única ou dupla e 2 anos para cardioversor-desfibrilador implantável (CDI). ${ }^{14}$

Em nosso meio, há escassa informação sobre esse tema, além do fato de as características dos pacientes e as etiologias para o implante de DCEI serem distintas dos países desenvolvidos. Portanto, o conhecimento do perfil desses pacientes e sua evolução são etapas iniciais importantes para a execução das diretrizes estabelecidas na literatura. ${ }^{15}$ À vista disso, os objetivos deste estudo foram verificar a proporção de pacientes com infecção relacionada a DCEI e analisar seu perfil clínico e laboratorial, as variáveis relacionadas com a infecção e sua evolução.

\section{Métodos}

Trata-se de um estudo de coorte, observacional, longitudinal e retrospectivo. A população foi constituída por 123 pacientes com infecção relacionada a DCEI, de ambos os sexos e de todas as idades, no período de 2001 a 2017, considerando 6.406 procedimentos de implante de DCEI. Foram excluídos pacientes com infecção relacionada a MP temporário, e também foram excluídos pacientes com infecção do DCEI, porém submetidos ao implante do dispositivo em outros hospitais. Tanto o projeto de pesquisa quanto o termo de consentimento livre e esclarecido foram aprovados pelo Comitê de Ética em Pesquisa da instituição, em conformidade com o descrito na resolução 466/2012. Foram analisadas variáveis clínicas e laboratoriais e dados de tratamento farmacológico e não farmacológico. Para o diagnóstico de infecção relacionada a DCEI, foram considerados exames clínicos associados a hemograma, proteína C-reativa, hemoculturas e ecocardiograma. Para diagnóstico de endocardite infecciosa, foram utilizados os critérios de Duke modificados. ${ }^{16}$

A profilaxia e o tratamento das infecções relacionadas a DCEI realizados na instituição na qual foi desenvolvido o estudo incluíram técnicas assépticas com banho de clorexidene dergemante na noite anterior e na manhã da realização do procedimento, remoção de pelo, degermação cirúrgica, antissepsia da pele com clorexidine degermante durante 2 minutos e, após remover o excesso, aplicação de clorexidine alcoólica. Segundo o mesmo protocolo, a profilaxia antibiótica foi realizada 1 hora antes do procedimento com dose única de cefazolina $2 \mathrm{~g}$.

O quadro de sepse foi definido como disfunção orgânica potencialmente fatal causada por uma resposta imune desregulada a uma infecção. ${ }^{17}$ Foram considerados mortalidade intra-hospitalar todos os óbitos ocorridos em razão da infecção no período de internação. Após a alta hospitalar, os pacientes sobreviventes foram acompanhados durante um período mínimo de 6 meses. Foram considerados morte após a alta hospitalar todos os óbitos naturais, fossem cardíacos ou não. Para a mortalidade total, foram considerados os óbitos intra-hospitalares por infecção relacionada a DCEI e os óbitos durante o seguimento clínico após a alta hospitalar.

\section{Análise Estatística}

Para a análise dos dados, foi utilizado o programa SPSS, versão 14.0. As variáveis categóricas foram expressas em números e proporção, e as variáveis contínuas foram expressas em médias e desvio padrão. Os testes do qui-quadrado e de Fisher, quando apropriados, foram utilizados para estudar associações entre variáveis categóricas. Para a comparação entre variáveis contínuas, foi utilizado o teste t de Student não pareado, em razão da distribuição normal por meio do teste de Kolmogorov-Smirnov. O intervalo de confiança (IC) foi de $95 \%$. Foi realizada análise da sobrevida por meio da curva de Kaplan-Meier. Foi aplicado o teste de log-rank para comparar as curvas de sobrevida. O nível de significância adotado na análise estatística foi de 5\%. 


\section{Resultados}

\section{Características Gerais da Casuística}

A média de idade dos 123 pacientes com infecção relacionada a DCEI foi de 60,1 $\pm 19,4$ anos (variando de 3 meses a 97 anos), sendo 71 (57,7\%) do sexo masculino. O número médio de procedimentos realizados, considerando implantes, trocas e manipulações de eletrodos, foi de 1,7. A média da fração de ejeção do ventrículo esquerdo (FEVE) foi de $48,4 \%$. Considerando o tempo de inclusão dos pacientes com infecção relacionada a DCEI, o qual foi de 16 anos, a taxa anual de infecção foi de 1,2 por 1.000 procedimentos. As principais cardiopatias de base são apresentadas na Figura 1. Em relação aos DCEl, o modo de estimulação foi MP em modo VVI em 38,2\%, em modo DDD em 30,9\%, em modo AAI em 2,4\%, CDI em 19,5\% e terapia de ressincronização cardíaca (TRC) em 9\% dos pacientes.

\section{Variáveis Relacionadas à Infecção}

Todos os pacientes apresentaram sinais e/ou sintomas sugestivos de infecção por DCEI. Foram observados secreção em bolsa em $39(31,7 \%)$ pacientes, febre e mal-estar generalizado em $23(18,6 \%)$ e bolsa com sinais de hiperemia e flutuação em 16 (13,0\%). Houve extrusão do gerador em $45(36,5 \%)$ pacientes.

Foram colhidas hemoculturas de todos os pacientes. $\mathrm{O}$ agente etiológico mais prevalente foi o estafilococo, isolado em culturas de 63 (51,2\%) pacientes, seguido por estreptococo, encontrado em 2 (1,6\%). Outros agentes, como serratia, pseudomnas aeruginosa, enterococcus faecalis e klebsiella, foram isolados em 20 (16,3\%) pacientes. Houve presença de mais de um agente etiológico em 36 (29,7\%) hemoculturas, e 38 (30,9\%) foram negativas.
Foram realizadas culturas da secreção da loja do gerador e da ponta de eletrodos em 74 pacientes. Entre os resultados da primeira, foram encontrados os agentes etiológicos $S$. aureus e $S$. epidermidis em 15 (20,2\%) e 5 (6,7\%) pacientes, respectivamente. Os demais agentes etiológicos, como pseudomonas, escherichia coli e acinetobacter baumannii, foram isolados em $8(10,8 \%)$ amostras. A cultura de ponta de cateter identificou os agentes etiológicos $S$. aureus e S. epidermidis em 21 (28,3\%) e 18 (24,3\%) pacientes, respectivamente. Os demais agentes, como serratia marcescens, pseudomonas e aeromonas hydrophila, foram isolados em 7 (9,4\%) amostras.

O ecocardiograma transesofágico foi realizado em 91 $(73,9 \%)$ pacientes, sendo que $44(35,7 \%)$ apresentaram imagem sugestiva de vegetação, enquanto o ecocardiograma transtorácico foi utilizado em 114 pacientes. Outros dados laboratoriais, como leucócitos, proteína C-reativa, intervalo de tempo entre o último implante e o diagnóstico de infecção e o tempo de internação, estão dispostos na Tabela 1.

Ocorreu infecção relacionada a DCEI causada pela realização do primeiro implante em $58(47,1 \%)$ pacientes. Em 55 (44,7\%), ocorreu devido à troca de gerador e, em 10 $(8,1 \%)$, por manipulações como plastia de loja de gerador, upgrade e reposicionamento de eletrodo. Houve infecção precoce (considerando o intervalo de tempo entre o procedimento e o diagnóstico de infecção inferior a 1 ano) em $78(63,4 \%)$ pacientes. Não houve influência das variáveis sexo, idade, índice de massa corporal, número de procedimentos, tipo de dispositivo e fração de ejeção.

\section{Abordagens Farmacológica e Não Farmacológica em Relação à Infecção}

O antibiótico mais utilizado foi a vancomicina [91 (73,9\%) pacientes], seguido pela oxacilina [20 (16,2\%) pacientes].

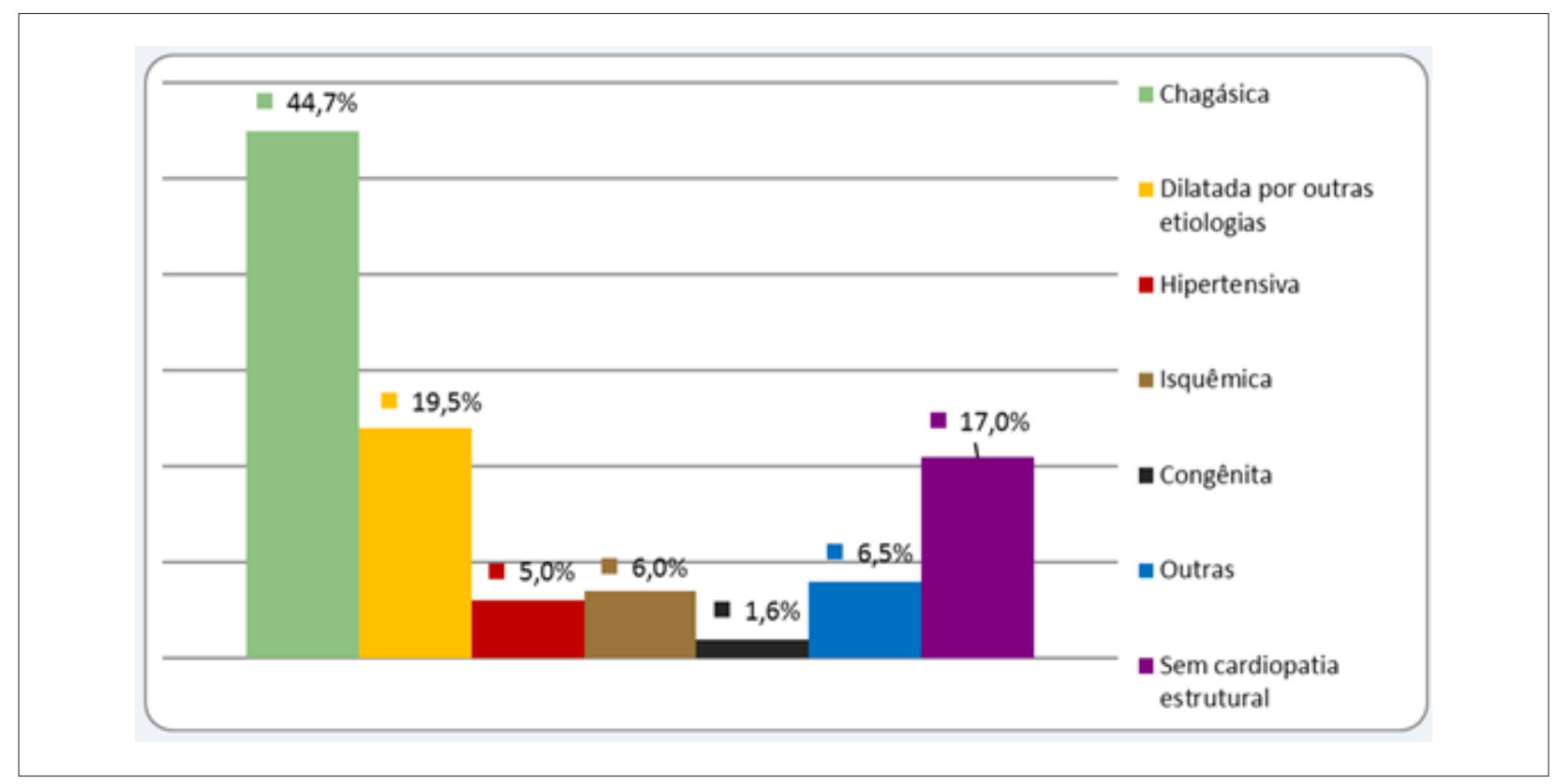

Figura 1 - Principais cardiopatias de base. 


\begin{tabular}{lccccc}
\hline \multicolumn{2}{l}{ Tabela 1 - Variáveis relacionadas à infecção } & & & & \\
\hline Variáveis & Média & Desvio padrão & Mínimo & Máximo & Mediana \\
\hline$\Delta$ tempo (dias) & 563,36 & 936,43 & 1 & 5895 & 138,5 \\
\hline Leucócitos $\left(\mathrm{ml} / \mathrm{mm}^{3}\right)$ & $9.502,7$ & $5.900,9$ & $1.008,0$ & $51.310,0$ & $8.350,0$ \\
\hline PCR (mg/L) & 68,7 & 81,3 & 3 & 376,6 & 34,3 \\
\hline Tempo de internação (dias) & 35,3 & 22,3 & 1 & 131 & 29,0 \\
\hline
\end{tabular}

tempo: intervalo de tempo entre o último implante e o diagnóstico de infecção; PCR: proteína C-reativa.

A retirada total do sistema foi feita em $105(85,4 \%)$ pacientes, enquanto a parcial foi feita em $11(8,9 \%)$. Foram tratados somente com antibióticos sete $(5,7 \%)$ pacientes. Entre aqueles que foram submetidos à retirada parcial, oito $(6,5 \%)$ apresentaram recidiva da infecção.

Foram submetidos a reimplante de novos sistemas 108 pacientes, sendo por via endocárdica em 64 (52\%) e por via epicárdica em 44 (35,7\%). O reimplante do DCEI não foi realizado em 15 pacientes (12,1\%) pelos seguintes motivos: quatro foram submetidos a transplante cardíaco, três faleceram antes do procedimento e a família de um dos pacientes não autorizou o reimplante. Em três pacientes, a equipe médica optou por não realizar o procedimento.

\section{Evolução Intra-hospitalar dos Pacientes}

A média do período de internação hospitalar foi de $35,3 \pm 22,3$ dias, variando de 1 a 131 dias. Houve evolução sem complicações no período da internação em 40 (32,5\%) pacientes. Houve piora da função renal em 37 (30,0\%) pacientes; tromboembolismo pulmonar, encefalopatia e meningite em 27 (21,9\%); derrame pleural em 11 (8,9\%); e necessidade de ventilação mecânica em oito $(6,5 \%)$. Ainda, 71 (57,7\%) pacientes evoluíram com endocardite infecciosa e, entre esses, 19 (15,4\%) evoluíram com sepse. Foi diagnosticada sepse em $23(18,7 \%)$ pacientes, sendo que $15(12,1 \%)$ faleceram em virtude disso. Em relação à endocardite e ao tipo de dispositivo, 55,6\% dos pacientes que cursaram com endocardite estavam com MP, 62,5\% com CDI e 54,5\% com TRC ( $p=0,65)$. Os demais dados sobre as variáveis associadas ou não a endocardite infecciosa estão dispostos na Tabela 2.

A mortalidade intra-hospitalar foi de 19,5\% (24 pacientes); todos os óbitos foram por infecção relacionada a DCEI. A comparação entre os pacientes que cursaram sem e com morte intra-hospitalar é apresentada na Tabela 3. O risco para morte intra-hospitalar em relação ao curso com endocardite infecciosa foi de 4,47 (com IC95\% entre 1,42 e 14,1). Quanto ao curso com sepse, o risco para morte intra-hospitalar foi de 4,1 (IC95\% entre 1,3 e 12,9). Em relação ao tipo de dispositivo, 18 (20,5\%) pacientes com MP, 4 com CDI (16,6\%) e 2 com TRC $(18,2 \%)$ faleceram no período intra-hospitalar $(p=0,42)$.

\section{Evolução Após a Alta Hospitalar}

Houve mais de uma infecção em oito pacientes. Foi dada alta hospitalar a 99 (80,4\%) pacientes, com seguimento clínico médio de 43,8 meses e mediana de 28,3, variando de 0,6 a 177 meses. A taxa de mortalidade após a alta hospitalar foi de $29,3 \%$ (29 pacientes), ocorrendo entre 3,94 a 164,5 meses.

\section{Curvas de Sobrevida}

Utilizando a curva de Kaplan-Meier e considerando como base prognóstica a ocorrência de morte total (por

Tabela 2 - Comparação das médias das variáveis entre os grupos de pacientes com e sem endocardite infecciosa

\begin{tabular}{lccc}
\hline Variáveis & $\begin{array}{c}\text { Grupo sem } \\
\text { endocardite }\end{array}$ & $\begin{array}{c}\text { Grupo com } \\
\text { endocardite }\end{array}$ & Valor-p* $^{*}$ \\
\hline Sexo masculino & $31(25,2 \%)$ & $39(31,7 \%)$ & 0,51 \\
\hline Idade (anos) & $60,2 \pm 18,9$ & $60,0 \pm 19,9$ & 0,95 \\
\hline IMC (kg/m2) & $24,5 \pm 5,1$ & $24,2 \pm 4,9$ & 0,77 \\
\hline Fração de ejeção (\%) & $45,0 \pm 16,4$ & $50,4 \pm 17,7$ & 0,99 \\
\hline Extrusão de gerador & $24(19,5 \%)$ & $21(17,0 \%)$ & 0,045 \\
\hline N de procedimentos & $1,6 \pm 0,8$ & $1,8 \pm 0,9$ & 0,405 \\
\hline Sepse & $4(3,2 \%)$ & $19(15,4 \%)$ & 0,010 \\
\hline Leucócitos $\left(\mathrm{ml}^{\prime} / \mathrm{mm}^{3}\right)$ & $8.638 \pm 9.886$ & $8.568 \pm 7.351$ & 0,96 \\
\hline PCR (mg/L) & $51,6 \pm 56,4$ & $80,9 \pm 93,6$ & 0,043 \\
\hline
\end{tabular}

IMC: índice de massa corpórea; PCR: proteína C-reativa; N: número. *Teste qui-quadrado ou Fisher ou teste $t$ de Student não pareado. 


\begin{tabular}{|c|c|c|c|}
\hline Variáveis & $\begin{array}{l}\text { Grupo sem morte intra- } \\
\text { hospitalar }(n=99)\end{array}$ & $\begin{array}{c}\text { Grupo com morte } \\
\text { intra-hospitalar }(n=24)\end{array}$ & Valor-p* \\
\hline Sexo masculino & $57(46,4 \%)$ & $14(11,3 \%)$ & 0,94 \\
\hline Idade (anos) & $59,9 \pm 18,6$ & $61,2 \pm 22,8$ & 0,79 \\
\hline IMC $\left(\mathrm{kg} / \mathrm{m}^{2}\right)$ & $24,7 \pm 4,9$ & $22,9 \pm 5,9$ & 0,21 \\
\hline Fração de ejeção (\%) & $49,0 \pm 17,3$ & $45,9 \pm 17,9$ & 0,45 \\
\hline $\mathrm{N}$ de procedimentos prévios & $1,73 \pm 0,9$ & $1,95 \pm 0,9$ & 0,317 \\
\hline Proporção de pacientes que cursaram com El & 52,0 & 83,3 & 0,005 \\
\hline Proporção de pacientes que cursaram com sepse & 8,1 & 62,5 & $<0,0001$ \\
\hline Leucócitos $\left(\mathrm{ml} / \mathrm{mm}^{3}\right)$ & $8.580 \pm 8.646$ & $8.661 \pm 7.777$ & 0,96 \\
\hline PCR (mg/L) & $62,73 \pm 72,0$ & $94,76 \pm 111,4$ & 0,22 \\
\hline
\end{tabular}

El: endocardite infecciosa; IMC: índice de massa corporal; N: número; PCR: proteína C-reativa. *Teste qui-quadrado ou Fisher ou teste $t$ de Student não pareado.

causa cardíaca e não cardíaca), foram construídas curvas de sobrevida. O teste de log-rank (Mantel-Cox) foi aplicado para comparar as curvas.

\section{- Sobrevida Total}

Durante todo o seguimento clínico de 43,8 meses, $53(43,0 \%)$ pacientes faleceram, sendo 24 no período intra-hopitalar e 29 após a alta hospitalar. A taxa anual de mortalidade total foi $11,8 \%$ e 0,52 por 1.000 procedimentos/ ano. A curva de sobrevida total dessa casuística é apresentada na Figura 2.

\section{- Sepse}

Dos 23 pacientes diagnosticados com sepse, 15 (65,2\%) faleceram durante o seguimento de 43,8 meses, com $\mathrm{p}<0,0001$ pelo teste de log-rank (Figura 3). A análise com seguimento de 6 e 36 meses apresentou o mesmo valor-p.

\section{- Outras Variáveis}

Não houve diferença na sobrevida quanto ao sexo $(p=0,89)$ e ao agente etiológico $(p=0,11)$. Em relação ao tipo de dispositivo, a taxa de mortalidade foi de $48,8 \%$ entre os pacientes com MP, 29,2\% entre os pacientes com CDI e $27,2 \%$ entre aqueles com TRC ( $p=0,92)$. Entre os pacientes que apresentaram endocardite durante a internação, 47,8\% faleceram durante o seguimento clínico de 43,8 meses ( $p=0,93$ ), sem diferença significativa, inclusive com seguimento de 6 e 36 meses $(p=0,11$ e 0,08 , respectivamente). Considerando a fração de ejeção $<50 \%$ e $\geq 50 \%$, a taxa de mortalidade foi de $44,2 \%$ e $41,5 \%$, respectivamente, durante todo o seguimento clínico $(\mathrm{p}=0,06)$. Quanto à modalidade de tratamento, faleceram $42,8 \%$ dos pacientes tratados somente com antibioticoterapia, $18,2 \%$ dos submetidos à retirada parcial do sistema e 47,7\% dos submetidos à retirada total do sistema $(p=0,07)$. Tomando por base prognóstica o tipo de reimplante, 32,8\% dos pacientes submetidos ao implante endocárdico e 52,2\% daqueles submetidos ao epicárdico faleceram $(p=0,04)$.

\section{Comparação entre Chagásicos e Não Chagásicos}

Na comparação entre pacientes chagásicos e não chagásicos, não houve diferença quanto às variáveis (idade, sexo, tipo de dispositivo, número de procedimentos, intervalo entre o último implante e o diagnóstico de infecção, valores de leucócitos, proteína C-reativa, FEVE, tempo de internação, agente infeccioso, presença de extrusão do gerador, proporção de endocardite infecciosa e de sepse e modalidade de tratamento) nem quanto à mortalidade (intra-hospitalar e após a alta).

\section{Discussão}

O implante de DCEI aumentou de forma significativa ao longo dos últimos anos devido à maior abrangência das indicações desses dispositivos e em virtude do aumento da expectativa de vida e do maior número de cardiopatas. A infecção relacionada a DCEI constitui um grave problema, com elevados índices de morbidade e mortalidade e com grande impacto socioeconômico em razão de seu alto custo de tratamento. ${ }^{7,18,19}$ No presente estudo, a média da idade dos pacientes foi semelhante à observada em outros estudos, ${ }^{9,13}$ assim como o predomínio do sexo masculino entre os pacientes com infecção relacionada a DCEI. ${ }^{9,20,21} \mathrm{~A}$ etiologia principal da cardiopatia de base no presente estudo, a chagásica, foi distinta da de outros países, nos quais há prevalência da cardiopatia isquêmica. ${ }^{4,9}$

As taxas de infecção podem variar dependendo da duração do seguimento e do tipo de dispositivo e de procedimento. ,21 $^{5}$ Este estudo revelou que houve maior proporção de infecção relacionada a procedimentos de troca de gerador, upgrade e plastia de bolsa. O tempo entre a última manipulação e o diagnóstico de infecção na literatura foi, em média, de 20 meses, ${ }^{13}$ semelhante ao presente estudo, mas pode variar dependendo do dispositivo, com intervalo de 4,2 meses 


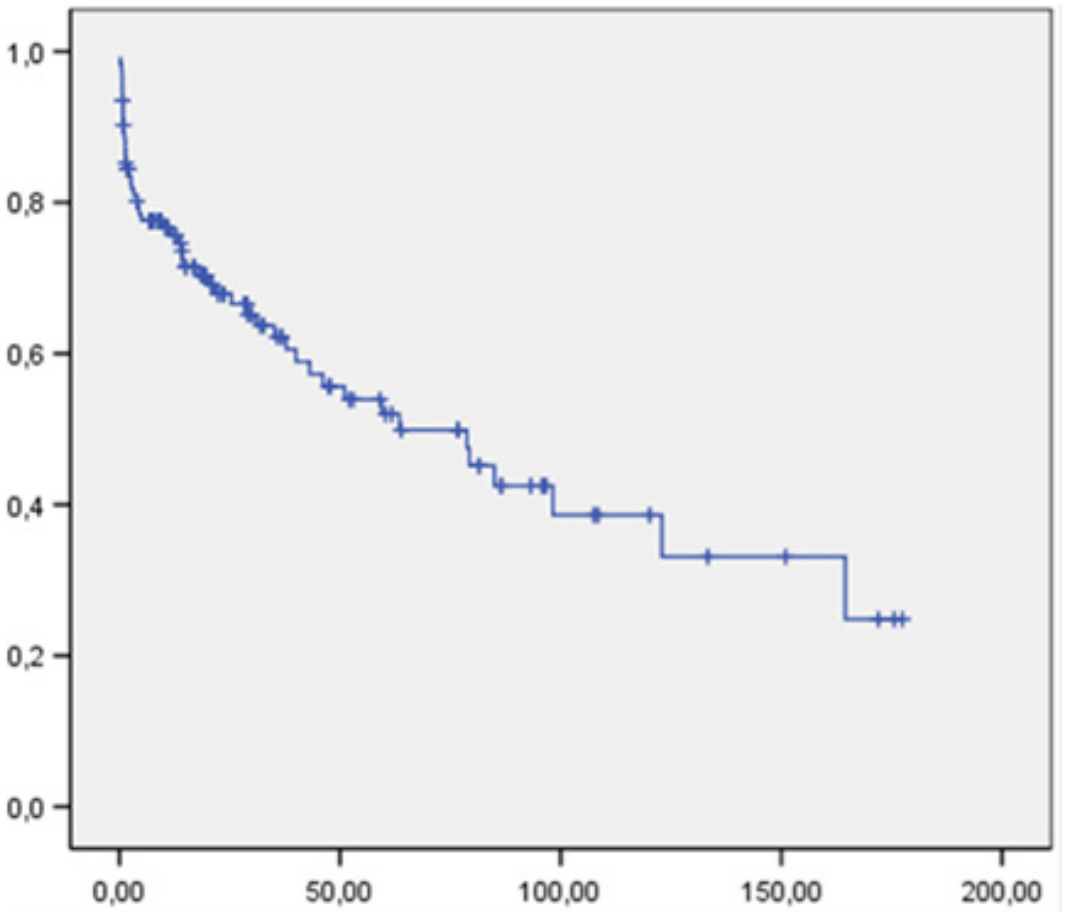

Figura 2 - Curva de sobrevida de toda a casuística. \%"Eixo horizontal: tempo em meses; eixo vertical: probabilidade acumulada de sobrevida \%.

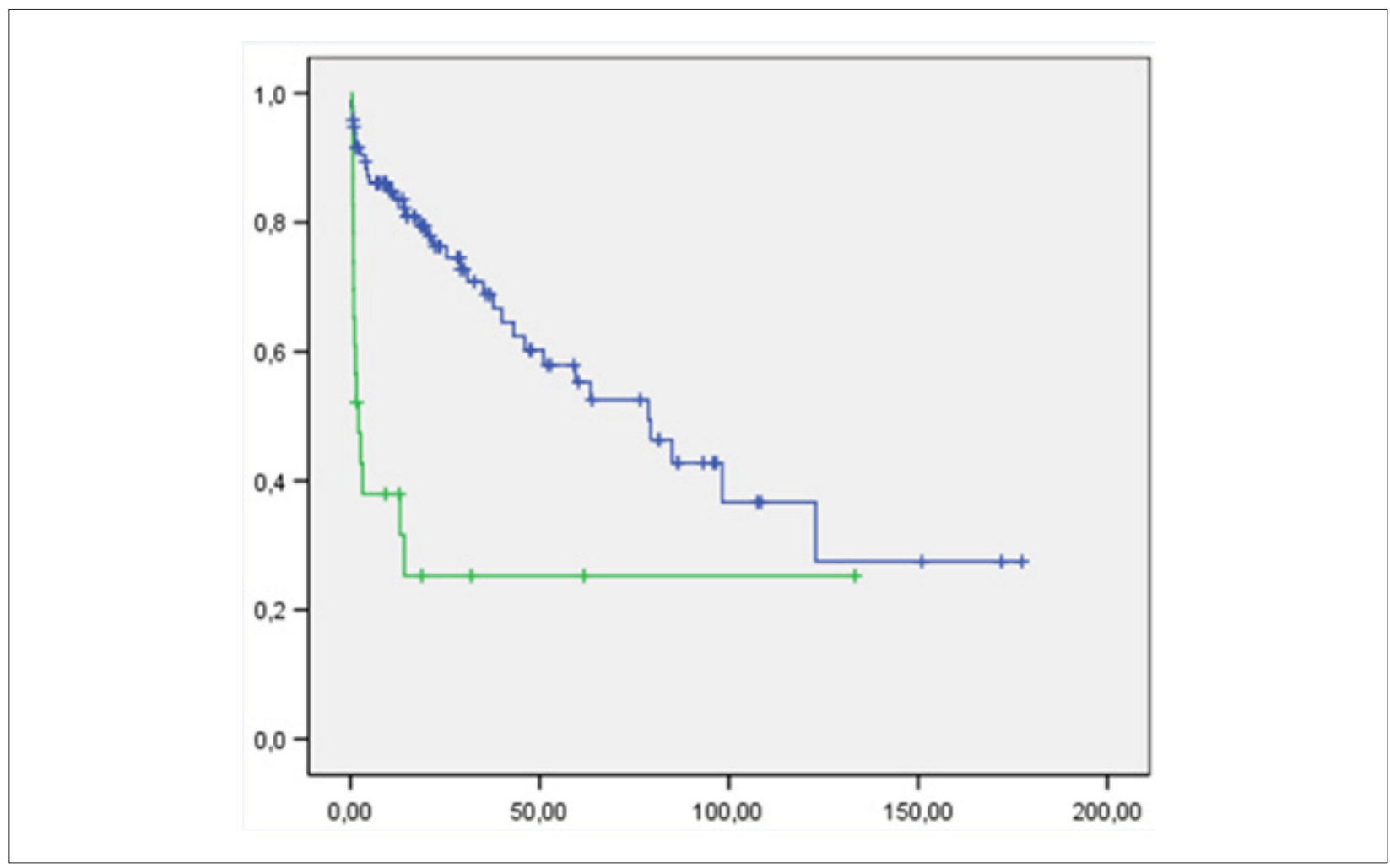

Figura 3 - Curva de sobrevida em relação à sepse. Eixo horizontal: tempo em meses; eixo vertical: probabilidade acumulada de sobrevida. Curva azul: pacientes que cursaram sem sepse durante a internação; curva verde: pacientes que cursaram com sepse. 
em relação ao $\mathrm{CDI}^{22}{ }^{22}$ Quanto à etiologia da infecção, as espécies de estafilococos causam a maior parte das infecções relacionadas a DCEI, sendo responsáveis por $60 \%$ a $80 \%$ dos casos relatados, ${ }^{1,23}$ taxa maior que a do presente estudo. Contudo, houve alta taxa de hemoculturas negativas, diferindo do reportado na literatura. ${ }^{1,5,24}$ Essa discordância pode ser atribuída ao uso prévio de antibióticos pelos pacientes, antes de seu ingresso no hospital. ${ }^{25}$

Para o diagnóstico da infecção relacionada com DCEI, além do método clínico, estão indicados exames laboratoriais e ecocardiograma. O ecocardiograma transesofágico é o mais indicado para o diagnóstico de infecção endovascular em razão de sua sensibilidade de $88 \%$ e especificidade de $99 \%$. Já o ecocardiograma transtorácico apresenta sensibilidade de apenas $32 \%$. ${ }^{3}$ Portanto, apesar da alta sensibilidade do exame e de sua indicação precisa nesse quadro, a correlação clínica e os resultados de hemoculturas são fundamentais para o diagnóstico e para as complicações referentes à essa infecção.

Uma dessas complicações é a endocardite, infecção grave que pode ocorrer entre 0,06 a 7,0\% dos casos de infecção relacionada a DCEI. ${ }^{13}$ Apresenta incidência anual de 1,83 casos/milhão de habitantes e 390 casos/milhão de portadores de $\mathrm{MP}^{26}$ e mortalidade relatada de até $26 \% .{ }^{27} \mathrm{Na}$ população estudada, 57,7\% dos pacientes desenvolveram endocardite. Em concordância com a literatura, o presente estudo mostrou o agravamento do prognóstico daqueles que cursaram com endocardite. Outra complicação foi a sepse, também contribuindo para o elevado número de óbitos, cuja taxa, segundo a literatura, pode variar de 32,2 a 51,1\%, tendo como principal agente o $S$. aureus. ${ }^{28,29}$ A tendência de associação inversa entre endocardite e extrusão do gerador no presente estudo pode ser explicada pelo número de pacientes com endocardite e extrusão, resultando em um viés de confusão, uma vez que o sinal extrusão pode ou não estar presente nos casos de endocardite.

Estudos recomendam o uso da vancomicina como prioritária no início da terapia antibiótica empírica no tratamento da infecção relacionada a DCEl, até que sejam verificados os resultados das hemoculturas. ${ }^{1} \mathrm{Em}$ concordância com a literatura, no estudo em questão, a vancomicina foi utilizada em $73,9 \%$ dos casos. Além da terapia com antibióticos, há outras modalidades adicionais de tratamento, como a retirada precoce e total do sistema do dispositivo com impacto favorável na evolução dos pacientes, associada a melhor sobrevida. ${ }^{11}$ Foram observados, no presente estudo, os benefícios da realização da retirada total do sistema de DCEI, com o objetivo de cura da infecção sem recidiva. Entretanto, a retirada total do sistema envolve, às vezes, cirurgia mais complexa como a cardiotomia, que pode agravar o quadro dos pacientes em questão. Dados da literatura demonstraram que a rápida extração do dispositivo e de seus eletrodos, associada a uma antibioticoterapia adequada com reimplante de novo dispositivo epicárdico ou contralateral, resultou em elevado índice de cura, com baixo risco de mortalidade operatória e infecção recorrente. ${ }^{30}$ A técnica percutânea de extração de eletrodos apresenta menor taxa de risco, entretanto, a mortalidade pode atingir 1,2\% em centros experientes devido a sangramentos, perfurações vasculares e tamponamento cardíaco. ${ }^{31}$
A infecção relacionada a DCEI pode resultar em um tempo prolongado de internação, o qual é estendido por mais de 13\% em relação ao período de internação para o implante de novo dispositivo. ${ }^{32} \mathrm{O}$ tratamento com antibióticos, o procedimento de extração e de reimplante e as complicações associadas contribuem para esse aumento no tempo de internação, com impacto, também, econômico. O tempo médio de internação no presente estudo foi de 35,5 dias, enquanto nos relatos da literatura foi de 17 dias. ${ }^{22}$ Essa diferença pode ser explicada pela maior proporção de pacientes com endocardite infecciosa neste estudo, resultando em tratamento mais extenso com antibióticos, conforme preconizado pela literatura. ${ }^{3,15}$

Além da morbidade, a infecção relacionada a DCEI também apresenta mortalidade tanto intra-hospitalar quanto pós-alta hospitalar. A mortalidade intra-hospitalar demonstrou uma variação ampla, segundo a literatura, dependendo do número de pacientes, da idade avançada e da presença de comorbidades e de complicações durante o tratamento, com taxas entre 6 e 14\%. Além disso, apresentou mortalidade total de aproximadamente $20 \%$ em um ano, ${ }^{1,6,9}$ atingindo $26,9 \%$ durante o seguimento de 5 anos. ${ }^{1,8,33} \mathrm{Na}$ população estudada, a taxa de mortalidade intra-hospitalar foi maior do que as demostradas nos estudos citados, o que pode ser justificado pelo maior número de pacientes que desenvolveram endocardite e sepse. Em relação ao período após a alta hospitalar, estudos com seguimento de até 2 anos evidenciaram que a taxa de total de mortes pode ser considerável e variar de $6 \%$ a $35 \% .{ }^{34,35}$ No presente estudo, a taxa de mortalidade após a alta hospitalar foi de 23,5\% durante o seguimento de 43,8 meses, com taxa anual de $14,5 \%$, dentro da faixa de taxas descritas na literatura.

Como já descrito previamente, há variáveis associadas a desfechos desfavoráveis e preditores de mortalidade. ${ }^{1,10-13,21}$ No estudo em questão, não houve associação significativa, pelas curvas de Kaplan-Meier, entre a sobrevida e o tipo de dispositivo, o curso de endocardite infecciosa durante a internação e a modalidade de tratamento. Entretanto, houve diferença significativa em relação à complicação de sepse durante a internação, com menor sobrevida após a alta, assim como entre aqueles submetidos ao implante epicárdico.

Em relação à modalidade de tratamento, Kim et al., ${ }^{9}$ relataram que pacientes tratados de forma conservadora, ou seja, somente com antibioticoterapia, apresentaram taxa de óbito elevada em tempo com média de 25 dias. ${ }^{9}$ Também há estudos relatando que a remoção precoce do dispositivo esteve associada a maior sobrevida dos pacientes. ${ }^{2,36}$ Quando não há remoção total do dispositivo, a mortalidade pode aumentar em 7 vezes dentro de 30 dias. $^{3}$ Uma publicação atual, com 6.859 pacientes sem infecção relacionada a DCEI, comparando a evolução entre aqueles submetidos à extração e aqueles com abandono dos eletrodos, demonstrou que a remoção dos eletrodos foi associada a menor taxa de infecção durante o período de 5 anos, porém não houve impacto sobre a sobrevida dos pacientes. ${ }^{37}$ De maneira correlata, porém com população incluindo pacientes com infecção relacionada a DCEI, um estudo de caso-controle demonstrou taxas similares de mortalidade em pacientes com e sem infecção. ${ }^{34}$ Isso reflete a heterogeneidade das casuísticas dos estudos quanto ao perfil clínico, tempo de diagnóstico e de intervenção e 
quanto às comorbidades, variáveis que interferem, também, na sobrevida. Além disso, uma pesquisa publicada em junho de 2019, com a participação de 62 países, demonstrou que somente $39,9 \%$ dos profissionais que realizam o implante de DCEI fazem irrigação da bolsa com antibiótico e somente 44\% administram antibiótico profilático, com remoção completa do sistema em $62 \%$ das vezes em caso de infecção, ${ }^{38}$ o que ilustra a disparidade de abordagem em pacientes com infecção relacionadas ao DCEI.

Quanto ao implante epicárdico, um estudo comparando o reimplante de eletrodos de MP após a infecção demonstrou que os 65 pacientes submetidos a acesso epicárdico apresentaram um risco de endocardite tardia ou reintervenção do dispositivo 3,6 vezes maior do que os 37 pacientes submetidos a MP temporário e posterior reimplante endocárdico. ${ }^{39}$ Isso foi explicado pelas complicações associadas ao reimplante epicárdico.

A etiologia da cardiopatia de pacientes com DCEl influencia sua evolução. O prognóstico de pacientes com cardiopatia chagásica crônica é desfavorável quando comparado ao de outras etiologias. ${ }^{40} \mathrm{Na}$ literatura, não há estudos específicos sobre infecção relacionada a DCEI e etiologia chagásica, exceto um estudo sobre o diagnóstico microbiano com a cultura de fluidos. ${ }^{41}$ Comparando 15 pacientes com infecção e 68 sem infecção relacionada a DCEI, com um total de 19 chagásicos, não houve diferença entre os grupos quanto a essa etiologia. No presente estudo, com um total de 55 chagásicos, a comparação entre pacientes chagásicos e não chagásicos não demonstrou diferença entre as variáveis e quanto à evolução.

\section{Limitações do Estudo}

A parte retrospectiva do estudo foi uma desvantagem devido à menor disponibilidade de registros adequados nos prontuários, além da subnotificação de pacientes com infecção relacionada a DCEI. Isso pode ter repercutido na taxa de infecção, com algum viés na análise. Além disso, em virtude do longo tempo de inclusão dos pacientes, as técnicas e os aparelhos de ecocardiograma foram distintos durante o período, sem uniformidade de sua realização, impedindo a verificação da valva acometida nos casos de endocardite infecciosa. Como nem todos os pacientes foram submetidos ao ecocardiograma transesofágico, a taxa de endocardite pode ter sido subestimada.

\section{Conclusões}

A taxa de infecção foi de 1,9\% (1,2 por 1.000 procedimentos/ ano), com predomínio em homens e em pacientes com miocardiopatia dilatada. Durante a internação, a incidência de endocardite infecciosa foi de $57,7 \%$ e a de sepse, $18,7 \%$. Foi feita remoção total do sistema na maioria dos pacientes $(85,4 \%)$. A taxa de mortalidade intra-hospitalar foi de $19,5 \%$ e houve associação com endocardite e sepse. Após a alta hospitalar, a taxa de mortalidade anual foi de 11,8\%, com influência somente da ocorrência de sepse durante a internação e do implante epicárdico.

\section{Contribuição dos Autores}

Concepção e desenho da pesquisa, Obtenção de dados, Análise e interpretação dos dados e Redação do manuscrito: Maciel AS, Silva RMFL; Análise estatística e Revisão crítica do manuscrito quanto ao conteúdo intelectual importante: Silva RMFL.

\section{Potencial Conflito de Interesses}

Os autores declaram não haver conflito de interesses pertinentes.

\section{Fontes de Financiamento}

O presente estudo não contou com fontes de financiamento externas.

\section{Vinculação Acadêmica}

Este artigo é parte de dissertação de Mestrado de Alessandra de Souza Maciel pelo Programa de Pós-graduação em Ciências Aplicadas à Saúde do Adulto, Faculdade de Medicina de Minas Gerais.

\section{Referências}

1. Baddour LM, Epstein AE, Erickson CC, Knight BP, Levison ME, Lockhart PB, et al. Update on cardiovascular implantable electronic device infections and their management: a scientific statement from the American Heart Association. Circulation. 2010;121(3):458-77.

2. Athan E, Chu VH, Tattevin P, Selton-Suty C, Jones P, Naber C, et al. Clinical characteristics and outcome of infective endocarditis involving implantable cardiac devices. JAMA. 2012;307(16):1727-35.

3. Kusumoto FM, Schoenfeld MH, Wilkoff BL, Berul Cl, BirgersdotterGreen UM, Carrillo R, et al. 2017 HRS expert consensus statement on cardiovascular implantable electronic device lead management and extraction. Heart Rhythm. 2017;14(12):e503-e551.

4. Gutiérrez Carretero E, Arana Rueda E, Lomas Cabezas JM, Laviana Martínez F, Villa Gil-Ortega M, Acosta Martínez J, et al. Infections in Cardiac Implantable Electronic Devices: Diagnosis and Management in a Referral Center. Rev Esp Cardiol (Engl Ed). 2017;70(5):355-62.
5. Nielsen JC, Gerdes JC, Varma N. Infected cardiac-implantable electronic devices: prevention, diagnosis, and treatment. Eur Heart J. 2015;36(37):2484-90.

6. Baman TS, Gupta SK, Valle JA, Yamada E. Risk factors for mortality in patients with cardiac device-related infection. Circ Arrhythm Electrophysiol. 2009;2(2):129-34.

7. Greenspon AJ, Patel JD, Lau E, Ochoa JA, Frisch DR, Ho RT, et al. 16-year trends in the infection burden for pacemakers and implantable cardioverterdefibrillators in the United States 1993 to 2008. J Am Coll Cardiol. 2011;58(10):1001-6.

8. Johansen JB, Jørgensen OD, Møller M, Arnsbo P, Mortensen PT, Nielsen JC. Infection after pacemaker implantation: infection rates and risk factors associated with infection in a population-based cohort study of 46299 consecutive patients. Eur Heart J. 2011;32(8):991-8. 
9. Kim DH, Tate J, Dresen WF, Papa FC, Bloch KC, Kalams SA, et al. Cardiac implanted electronic device-related infective endocarditis: clinical features, management, and outcomes of 80 consecutive patients. Pacing Clin Electrophysiol. 2014;37(8):978-85.

10. Uslan DZ, Dowsley TF, Sohail MR, Hayes DL, Friedman PA, Wilson WR, et al. Cardiovascular implantable electronic device infection in patients with Staphylococcus aureus bacteremia. Pacing Clin Electrophysiol. 2010;33(4):407-13.

11. Le KY, Sohail MR, Friedman PA, Uslan DZ, Cha SS, Hayes DL, et al. Impact of timing of device removal on mortality in patients with cardiovascular implantable electronic device infections. Heart Rhythm. 2011;8(11):1678-85.

12. Tarakji KG, Wazni OM, Harb S, Hsu A, Saliba W, Wilkoff BL. Risk factors for 1-year mortality among patients with cardiac implantable electronic device infection undergoing transvenous lead extraction: the impact of the infection type and the presence of vegetation on survival. Europace. 2014;16(10):1490-5.

13. Osmonov D, Ozcan KS, Erdinler I, Altay S, Yildirim E, Turkkan C, et al. Cardiac device-related endocarditis: 31-Years' experience. J Cardiol. 2013;61(2):175-80.

14. Rizwan Sohail M, Henrikson CA, Jo Braid-Forbes M, Forbes KF, Lerner DJ. Increased long-term mortality in patients with cardiovascular implantable electronic device infections. Pacing Clin Electrophysiol. 2015;38(2):231-9.

15. Sandoe JA, Barlow G, Chambers JB, Gammage M, Guleri A, Howard P, et al. Guidelines for the diagnosis, prevention and management of implantable cardiac electronic device infection. Report of a joint Working Party project on behalf of the British Society for Antimicrobial Chemotherapy (BSAC, host organization), British Heart Rhythm Society (BHRS), British Cardiovascular Society (BCS), British Heart Valve Society (BHVS) and British Society for Echocardiography (BSE). J Antimicrob Chemother. 2015;70(2):325-59.

16. Durack DT, Lukes AS, Bright DK. New criteria for diagnosis of infective endocarditis: utilization of specific echocardiographic findings. Duke Endocarditis Service. Am J Med. 1994;96(3):200-9.

17. Seymour CW, Liu VX, Iwashyna TJ, Brunkhorst FM, Rea TD, Scherag A, et al. Assessment of Clinical Criteria for Sepsis: For the Third International Consensus Definitions for Sepsis and Septic Shock (Sepsis-3). JAMA. 2016;315(8):762-74.

18. Lee WH, Huang TC, Lin LJ, Lee PT, Lin CC, Lee CH, et al. Efficacy of postoperative prophylactic antibiotics in reducing permanent pacem infections. Clin Cardiol. 2017; 40(8): 559-565.

19. Habib A, Le KY, Baddour LM, Friedman PA, Hayes DL, Lohse CM, et al. Predictors of mortality in patients with cardiovascular implantable electronic device infections. Am J Cardiol. 2013;111(6):874-9.

20. Wang R, Li X, Wang Q, Zhang Y, Wang H. Microbiological Characteristics and Clinical Features of Cardiac Implantable Electronic Device Infections at a Tertiary Hospital in China. Front Microbiol. 2017;8:360.

21. Olsen T, Jørgensen OD, Nielsen JC, Thøgersen AM, Philbert BT, Johansen JB. Incidence of device-related infection in 97750 patients: clinical data from the complete Danish device-cohort (1982-2018). Eur Heart J. 2019; 40(23):1862-1869

22. Sohail MR, Uslan DZ, Khan AH, Friedman PA, Hayes DL, Wilson WR, et al. Management and outcome of permanent pacemaker and implantable cardioverter-defibrillator infections. J Am Coll Cardiol. 2007;49(18):1851-9.

23. Klug D, Lacroix D, Savoye C, Goullard L, Grandmougin D, Hennequin JL, et al. Systemic infection related to endocarditis on pacemaker leads: clinical presentation and management. Circulation. 1997;95(8):2098-107.

24. Tarakji KG, Wilkoff BL. Cardiac implantable electronic device infections: facts, current practice, and the unanswered questions. Curr Infect Dis Rep. 2014;16(9):425.
25. Nof E, Epstein LM. Complications of cardiac implants: handling device infections. Eur Heart J. 2013;34(3):229-36.

26. Durval X, Seltn-Suty C, Salvador-Mazenq M, Bernard Y, Weber M,et al. Endocarditis in patients with a permanent pacemaker: a 2-year epidemiological survey on infective endocarditis due to valvular and/or pacemaker infection. Clin Infect Dis. 2004; 39(1):68-74.

27. Mansur AJ, Grinberg M,Cardoso RH, da Luz PL, Bellotti G, Pileggi F. Determinants of prognosis in 300 episodes of infective endocarditis. Thorac Cardiovasc Surg. 1996;44(1):2-10.

28. Hamid S, Arujuna A, Ginks M, McPhail M, Patel N, Bucknall C, et al. Pacemaker and defibrillator lead extraction: predictors of mortality during follow-up. Pacing Clin Electrophysiol. 2010;33(2):209-16.

29. VincentJL, Sakr Y, Sprung CL, Ranieri VM, Reinhart K, Gerlach H, etal. Sepsis in European intensive care units: results of the SOAP study. Crit Care Med. 2006;34(2):344-53.

30. Chua JD, Wilkoff BL, Lee I, Juratli N, Longworth DL, Gordon SM. Diagnosis and management of infections involving implantable electrophysiologic cardiac devices. Ann Intern Med. 2000;133(8):604-8.

31. Perez AA, Woo FW, Tsang DC, Carrillo RG. Transvenous Lead Extractions: Current Approaches and Future Trends. Arrhythm Electrophysiol Rev. 2018; 7(3):210-217.

32. Palmisano P, Accogli M, Zaccaria M, Luzzi G, Nacci F, Anaclerio M, etal. Rate, causes, and impact on patient outcome of implantable device complications requiring surgical revision: large population survey from two centres in Italy. Europace. 2013;15(4):531-40.

33. Greenspon AJ, Le KY, Prutkin JM, Sohail MR, Vikram HR, Baddour LM, et al. Influence of vegetation size on the clinical presentation and outcome of leadassociated endocarditis: results from the MEDIC registry. JACC CardiovasC Imaging. 2014;7(6):541-9.

34. Deharo JC, Quatre A, Mancini J, Khairy P, Le Dolley Y, Casalta JP, et al. Longterm outcomes following infection of cardiac implantable electronic devices: a prospective matched cohort study. Heart. 2012;98(9):724-31.

35. Leung S, Danik S. Prevention, Diagnosis, and Treatment of Cardiac Implantable Electronic Device Infections. Curr Cardiol Rep. 2016;18(6):58.

36. Viganego F, O'Donoghue S, Eldadah Z, Shah MH, Rastogi M, Mazel JA, et al. Effect of early diagnosis and treatment with percutaneous lead extraction on survival in patients with cardiac device infections. Am J Cardiol. 2012;109(10):1466-71.

37. Pokorney SD, Mi X, Lewis RK, Greiner M, Epstein LM, Carrillo RG, et al. Outcomes Associated With Extraction Versus Capping and Abandoning Pacing and Defibrillator Leads. Circulation.2017;136(15):1387-95.

38. Traykov V, Bongiorni MG, Boriani G, Burri H, Costa R, Dagres N, et al. Clinical practice and implementation of guidelines for the prevention, diagnosis and management of cardiac implantable electronic device infections: results of a worldwide survey under the auspices of the European Heart Rhythm Association. Europace. 2019; 21(8):1270-1279.

39. Perrin T, Maille B, Lemoine C, Resseguier N, Franceschi F, Koutbi L, et al. Comparison of epicardial vs. endocardial reimplantation in pacemakerdependent patients with device infection. Europace. 2018; 20(4):e42-e50.

40. Bocchi EA, Bestetti RB, Scanavacca MI, Cunha Neto E, Issa VS. Chronic Chagas Heart Disease Management: From Etiology to Cardiomyopathy Treatment. J Am Coll Cardiol. 2017; 70(12):1510-1524.

41. Inacio RC, Klautau GB, Murça MA, da Silva CB, Nigro S, Rivetti LA, et al. Microbial diagnosis of infection and colonization of cardiac implantable electronic devices by use of sonication. Int J Infect Dis. 2015; 38:54-9. 